\title{
Double-diffusive convection for a heated cylinder submerged in a salt-stratified fluid layer
}

\author{
Y.-M. Chen, C.-K. Liu
}

\begin{tabular}{|c|c|c|}
\hline Abstract Double-diffusive convection due to a cylindrical & $H$ & height of cavity $[\mathrm{m}]$ \\
\hline source submerged in a salt-stratified solution is numeri- & $J$ & Jacobian, $x_{\xi} y_{\eta}-x_{\eta} y_{\xi}$ \\
\hline cally investigated in this study. For proper simulation of & $L$ & width of cavity [m] \\
\hline $\begin{array}{l}\text { the vortex generated around the cylinder, a computational } \\
\text { domain with irregular shape is employed. Flow conditions }\end{array}$ & $L_{C L}$ & $\begin{array}{l}\text { length between the grid point at wall and which is } \\
\text { nearest the wall in Eq. (17) }\end{array}$ \\
\hline depend strongly on the thermal Rayleigh number, $R a_{T}$, & Le & Lewis number, $\alpha / D$ \\
\hline $\begin{array}{l}\text { and the buoyancy ratio, } R_{\rho} \text {. There are two types of onset of } \\
\text { instability existing in the flow field. Both types are due to }\end{array}$ & $N u, \overline{N u}$ & $\begin{array}{l}\text { local Nusselt number and average Nusselt number, } \\
\text { as defined in Eqs. (28) }\end{array}$ \\
\hline either the interaction of the upward temperature gradient & $P$ & dimensional pressure $\left[\mathrm{N} \mathrm{m}^{-2}\right]$ \\
\hline ownward salinity gradient or the interaction of the & $p$ & non-dimensional pressure, $P D^{2} / \rho \alpha^{2}$ \\
\hline lateral temperature gradient and downward salinity gra- & $\operatorname{Pr}$ & Prandtl number, $v / \alpha$ \\
\hline lient. The onset of layer instability due to plume con- & $R a_{T}$ & thermal Rayleigh number, $g \beta_{T} \Delta T D^{3} / \alpha v$ \\
\hline vection is due to the former, whereas, the onset of layer & $R a_{s}$ & solutal Rayleigh number, $g \beta_{S} \Delta T \eta^{3} / \alpha \nu$ \\
\hline nstability of layers around the cylinder is due to the latter. & $R_{\rho}$ & buoyancy ratio, $\beta_{S} \Delta C / \beta_{T} \Delta T$ \\
\hline 3oth types can be found in the flow field. The transport & $T$ & sional temperature $[\mathrm{K}]$ \\
\hline nechanism of layers at the top of the basic plume belongs & $T_{h}, T_{l}$ & highest and lowest temperature $[\mathrm{K}]$ \\
\hline o former while that due to basic plume and layer around & & sional time $[s]$ \\
\hline the cylinder are the latter. The increase in $R a_{T}$ reinforces & $U, V$ & sional velocities $\left[\mathrm{m} \mathrm{s}^{-1}\right]$ \\
\hline the plume convection and reduces the layer numbers & $U^{*}, V^{*}$ & defined in Eqs. (15) \\
\hline $\begin{array}{l}\text { generated around the cylinder for the same buoyancy ra- } \\
\text { tio. For the same } R a_{T} \text {, the increase of } R_{\rho} \text { suppresses the }\end{array}$ & $u, v$ & $\begin{array}{l}\text { dimensionless velocity in the } x \text {-direction, } \\
U D / \alpha, V D / \alpha\end{array}$ \\
\hline $\begin{array}{l}\text { plume convection but reinforces the layers generated } \\
\text { around the cylinder. The profiles of local Nusselt number }\end{array}$ & $X, Y$ & $\begin{array}{l}\text { dimensional coordinate in horizontal direction } \\
{[\mathrm{m}]}\end{array}$ \\
\hline $\begin{array}{l}\text { reflects the heat transfer characteristics of plume convec- } \\
\text { tion and layered structure. The profiles of averaged Nusselt }\end{array}$ & $x, y$ & $\begin{array}{l}\text { dimensionless coordinate in horizontal direction, } \\
X / D, Y / D\end{array}$ \\
\hline number are between the pure conduction and natural & $w_{f}(Z)$ & \\
\hline $\begin{array}{l}\text { convection modes and the variation is due to the evolution } \\
\text { of layers. }\end{array}$ & $w_{f}^{*}(Z)$ & $\begin{array}{l}\text { weighting functions defined in Eqs. (25) } \\
\text { parameter of the weighting functions. }\end{array}$ \\
\hline of syn & \multicolumn{2}{|c|}{ Greek symbols } \\
\hline a general function used in Eq. (22) & . & thermal diffusivity \\
\hline of discretized Eqs. (23) & & defined in Eqs. (15) \\
\hline$B$ & $\beta$ & geometry parameter defined in Eqs. (15) \\
\hline$C \quad$ dim & $\beta_{T}, \beta_{S}$ & volumetric coefficient of thermal expansion $\left[\mathrm{K}^{-1}\right]$ \\
\hline $\begin{array}{l}C_{h}, C_{l} \text { the highest and lowest concentration [wt\%] } \\
c \text { dimensionless solute, } C-C_{l} / C_{h}-C_{l}\end{array}$ & & or expansion with solutal $\left[\mathrm{wt}^{-1} \mathrm{H}^{-1}\right]$ \\
\hline$D \quad$ diameter of the cylinder [m] or mass & & angle around the cylinder \\
\hline used for Lewis number $\left[\mathrm{m}^{2} \mathrm{~s}^{-2}\right]$ & $\Delta$ & initial concentration difference, $C_{h}-C_{l}$ [wt $\left.\%\right]$ \\
\hline gravity $\left[\mathrm{ms}^{-2}\right]$ & $\Delta T$ & initi \\
\hline & $\Delta \psi$ & difference of stream function \\
\hline & & \\
\hline Received on 13 September 1996 & & curvilinear coordinate \\
\hline & & ord \\
\hline Y.-M. Chen, C.-K. Liu & & \\
\hline tment of Mechanical Enginee & & \\
\hline National Taiwan University, Taipei, & $(0$ & physical property of int \\
\hline Taiwan 10764, R.O.C. & $v$ & viscosity $\left[\mathrm{m}^{2} \mathrm{~s}^{-2}\right]$ \\
\hline & 0 & sionless temperature, $T$ \\
\hline Correspondence to & $\tau$ & dimensionless time, $t \alpha / L^{2}$ \\
\hline
\end{tabular}


$\psi \quad$ stream function, $u=\psi_{x}$ and $v=-\psi_{y}$.

$\psi_{C L} \quad$ stream function at the grid point which is nearest the wall

\section{Subscripts}

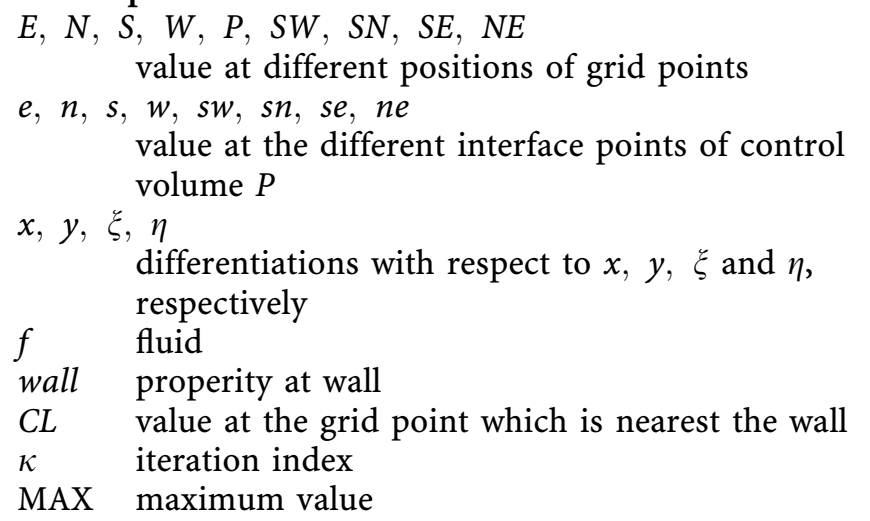

\section{Superscripts}

0 guessed value

\section{1}

\section{Introduction}

Double-diffusive phenomenon usually refers to a class of fluid motions that is subject to the simultaneous presence of two (or more) components with different molecular diffusivity [1]. One of the most striking features of doublediffusive phenomena is the formation of systems of layers separated by thin interfaces. This results from the interaction of the stable salinity gradient and the unstable temperature gradient [2]. Great interest has been taken in the occurrence of these phenomena in geophysical flows [1] and in engineering applications such as the salt-gradient solar pond, crystal growth and metal casting [2].

Some literature has been devoted to the study of the convection of layered systems due to heating the salt-stratified fluid from below by one- or two-dimensional analysis $[3,4]$. On the other hand, there is other kind of layered system that is due to salt-stratified fluid with lateral heating. Thorpe et al. [5] studied this kind of layers in a vertical slot with lateral heating first. Chen et al. [6] investigated, experimentally, the layered convection in a wide tank with lateral heating and the critical value of modified Rayleigh number was presented. If the modified Rayleigh number is larger than the critical Rayleigh number, the simultaneous layer structure can be seen at the middle of the sidewalls. Otherwise, only some successive layers can be seen near the top and bottom plates. Lee and Hyun [7] predicted these phenomena by numerical simulation. Pawail and Chen [8] made a study of layered convection in an inclined slot and they found the onset of instability of the layered system to be asymmetric. When the inclined angle is changed, the onset of instability is sooner for heating from above and is later for heating from below. Recently, Chen and Liu [9] studied the double-diffusive layered system in an inclined rectangular cavity. They showed that the directions of heating had significant influences on the layer formation, flow types and heat transfer characteristics.

On the other hand, for double-diffusive convection resulting from a circular heat source in the salt-stratified fluid, Hubbell and Gebhart [10] experimentally studied the formation of layers around a cylinder. They showed that there were layers around the cylinder and plume. The number of layers varies with different degrees of salt-stratified fluid. Tsinober et al. [11] further classified the layers into three different types by heating with a discrete heat source in a salt-stratified fluid. The first type is the layers above the plume. This phenomenon is due to heating from below. The second type develops around the upper part of the plume, and is driven by the same mechanism as the heating from the sidewall. The third type of layers form around the lower part of the plume, and are of the same nature as the second type but also have something in common with double-diffusive intrusions. Neilson and Incropera [12] conducted experiments on a cylindrical heat source with constant heat flux submerged in a salt-stratified fluid. They clearly describe the transport mechanism of all the types of layers. Dosch and Beer [13] numerically simulated the double-diffusive convection in a horizontal concentric annulus. Experiments on holographic visualization were also conducted in which they examined the third type of layer but the plume convection was not significant in their studies. They showed that the heat transfer characteristics of double-diffusive convection are between the modes of conduction and natural convection.

All of the above studies focused on the flow types of layers. The discussion of mechanisms of layer generation and transport phenomena in the three types of layers are still lacking, especially for the case of around a single cylinder, or cylinders inside a cavity. Therefore, double-diffusive convection due to submerging the heating cylinder in a salt-stratified rectangular cavity was numerically investigated in this study. Due to the special physical geometry of a rectangular shape outer surface and circular region inside, the body-fitted grid generation method was used to solve the problem. The developed grid generation method can be extended to the study of two or multicylinder cases. Focus was on the generation of layers and evolution of layered system due to plume convection and the lateral heating. The effects of thermal Rayleigh number and buoyancy ratio were also studied. The flow field, temperature and salinity distributions, as well as local and averaged Nusselt numbers are presented.

\section{2}

\section{Mathematical modeling}

Figure 1 depicts the geometry to be considered. A horizontal cylinder of diameter $D$ is submerged in a rectangular cavity with an aspect ratio of 3 . The distance between bottom of the cylinder and the bottom of the cavity is $0.5 \mathrm{D}$. The distance calculated from top of the cylinder to the top of cavity is $1.5 \mathrm{D}$. This geometry makes the plume convection less constrained. The flow is assumed to remain twodimensional, laminar and incompressible, with constant fluid properties, except for the density change due to temperature and salinity which drive the buoyancy force. This results in the use of the Boussinesq approximation. For simplication of the numerical solution, the flow is considered to be symmetrical with respect to the vertical 


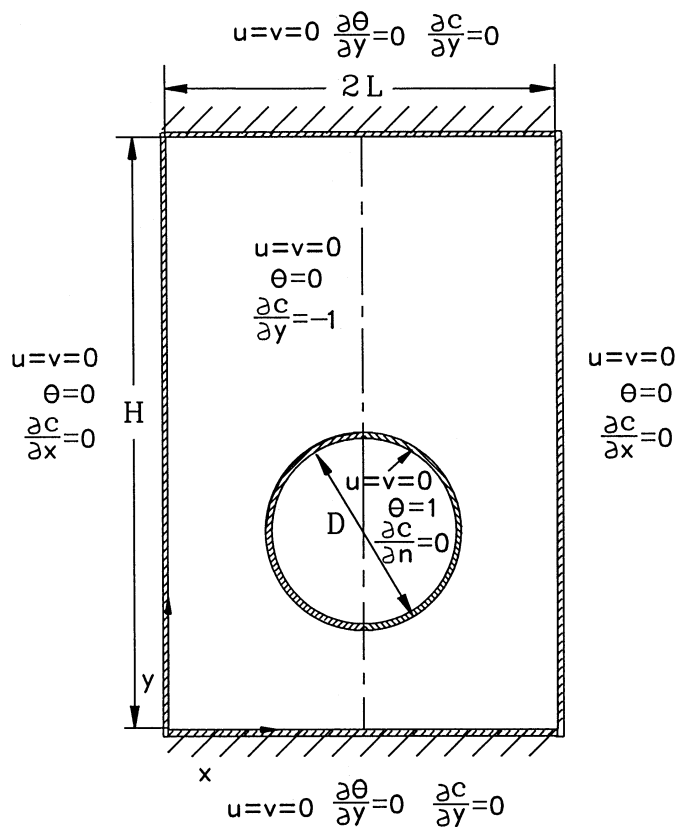

Fig. 1. Schematic of the physical domain, boundary conditions

central line. This assumption is valid when the height of plume convection is not very large, as demonstrated in the experimental results of $[10,12]$.

With the foregoing assumption, the governing equations of continuity, momentum, temperature and salinity can be written in the following dimensionless form.

$\frac{\partial u}{\partial x}+\frac{\partial v}{\partial y}=0$

$\frac{\partial u}{\partial \tau}+u \frac{\partial u}{\partial x}+v \frac{\partial u}{\partial y}=-\frac{\partial p}{\partial x}+\operatorname{Pr}\left(\frac{\partial^{2} u}{\partial x^{2}}+\frac{\partial^{2} u}{\partial y^{2}}\right)$

$\frac{\partial v}{\partial \tau}+u \frac{\partial v}{\partial x}+v \frac{\partial v}{\partial y}=-\frac{\partial p}{\partial y}+\operatorname{Pr}\left(\frac{\partial^{2} v}{\partial x^{2}}+\frac{\partial^{2} v}{\partial y^{2}}\right)$

$$
+\operatorname{PrRa}_{T}\left(\theta-R_{\rho} c\right)
$$

$\frac{\partial \theta}{\partial \tau}+u \frac{\partial \theta}{\partial x}+v \frac{\partial \theta}{\partial y}=\frac{\partial^{2} \theta}{\partial x^{2}}+\frac{\partial^{2} \theta}{\partial y^{2}}$

$\frac{\partial c}{\partial \tau}+u \frac{\partial c}{\partial x}+v \frac{\partial c}{\partial y}=\frac{1}{L e}\left(\frac{\partial^{2} c}{\partial x^{2}}+\frac{\partial^{2} c}{\partial y^{2}}\right)$.

In the above equations, the dimensionless variants are defined as

$x=\frac{X}{D}, y=\frac{Y}{D}, u=\frac{U D}{\alpha}, v=\frac{V D}{\alpha}, p=\frac{P D^{2}}{\rho \alpha^{2}}, \tau=\frac{t \alpha}{D^{2}}$

$\operatorname{Pr}=\frac{v}{\alpha}, R a_{T}=\frac{g \beta_{T}\left(T_{h}-T_{l}\right) D^{3}}{v \alpha}, R a_{S}=\frac{g \beta_{S}\left(C_{h}-C_{l}\right) D^{4}}{v \alpha \mathscr{H}}$

$R_{\rho}=\frac{R a_{S}}{R a_{T}}, \theta=\frac{T-T_{l}}{T_{h}-T_{l}}, c=\frac{C-C_{l}}{C_{h}-C_{l}}, L e=\frac{v}{D_{f}}$.

The appropriate boundary and initial conditions are shown in Fig. 1.

The stream function and vorticity are defined as $u=\frac{\partial \psi}{\partial y}, v=-\frac{\partial \psi}{\partial x} ; \omega=\frac{\partial v}{\partial x}-\frac{\partial u}{\partial y}$.

The next step is to generate a body-fitted coordinate system $(\xi, \eta)$, with a uniform step size $\Delta \xi=\Delta \eta=1$, by using the Poisson grid generation method proposed in Ref. [14]. The C-type grid mesh is used for solving the physical domain. Through coordinate transformation from the xyplane to the $\xi \eta$-plane, the physical domain is mapped to the rectangular computational region $0 \leq \xi \leq \xi_{M A X}$ and $0 \leq \eta \leq \eta_{\text {MAX }}$. The vertical central line and the surface of cylinder correspond to the right hand side of the rectangle $\left(\eta=\eta_{M A X}, 0 \leq \xi \leq \xi_{M A X}\right)$, while the upper and lower surfaces are the top $\left(\xi=\xi_{M A X}, 0 \leq \eta \leq \eta_{M A X}\right)$ and bottom $\left(\xi=0,0 \leq \eta \leq \eta_{\text {MAX }}\right)$ boundaries of the computational domain, respectively.

On the curvilinear coordinate system $(\xi, \eta)$, after inducing Eq. (6) on to the governing equations (1) to (5), they become as follows.

$$
\begin{gathered}
\frac{\partial}{\partial \xi}\left(\frac{\alpha}{J} \frac{\partial \psi}{\partial \xi}\right)+\frac{\partial}{\partial \eta}\left(\frac{\gamma}{J} \frac{\partial \psi}{\partial \eta}\right)-\frac{\partial}{\partial \xi}\left(\frac{\beta}{J} \frac{\partial \psi}{\partial \eta}\right) \\
-\frac{\partial}{\partial \eta}\left(\frac{\beta}{J} \frac{\partial \psi}{\partial \xi}\right)=-J \omega \\
J \frac{\partial \omega}{\partial \tau}+\frac{\partial}{\partial \xi}\left(U^{*} \omega\right)+\frac{\partial}{\partial \eta}\left(V^{*} \omega\right)=\frac{\partial}{\partial \xi}\left(\operatorname{Pr} \frac{\alpha}{J} \frac{\partial \omega}{\partial \xi}\right) \\
-\frac{\partial}{\partial \xi}\left(\operatorname{Pr} \frac{\gamma}{J} \frac{\partial \omega}{\partial \eta}\right)+\frac{\partial}{\partial \xi}\left(\operatorname{Pr} \frac{\beta}{J} \frac{\partial \omega}{\partial \eta}\right)-\frac{\partial}{\partial \eta}\left(\operatorname{Pr} \frac{\beta}{J} \frac{\partial \omega}{\partial \xi}\right) \\
\left.+\operatorname{Pr} \operatorname{Ra} \frac{\partial Y}{\partial \eta} \frac{\partial \theta}{\partial \xi}-\frac{\partial Y}{\partial \xi} \frac{\partial \theta}{\partial \eta}-R\left(\frac{\partial Y}{\partial \eta} \frac{\partial c}{\partial \xi}-\frac{\partial Y}{\partial \xi} \frac{\partial c}{\partial \eta}\right)\right] \\
J \frac{\partial \theta}{\partial \tau}+\frac{\partial}{\partial \xi}\left(U^{*} \theta\right)+\frac{\partial}{\partial \eta}\left(V^{*} \theta\right)=\frac{\partial}{\partial \xi}\left(\frac{\alpha}{J} \frac{\partial \theta}{\partial \xi}\right) \\
+\frac{\partial}{\partial \eta}\left(\frac{\gamma}{J} \frac{\partial \theta}{\partial \eta}\right)-\frac{\partial}{\partial \xi}\left(\frac{\beta}{J} \frac{\partial \theta}{\partial \eta}\right)-\frac{\partial}{\partial \eta}\left(\frac{\beta}{J} \frac{\partial \theta}{\partial \xi}\right) \\
+\frac{\partial}{\partial \eta}\left(\frac{1}{L e} \frac{\gamma}{J} \frac{\partial c}{\partial \eta}\right)-\frac{\partial}{\partial \xi}\left(\frac{1}{L e} \frac{\partial c}{J} \frac{\partial c}{\partial \eta}\right) \\
J \frac{\partial}{\partial \tau}+\frac{\partial}{\partial \xi}\left(U^{*} c\right)+\frac{\partial}{\partial \eta}\left(V^{*} c\right)=\frac{\partial}{\partial \xi}\left(\frac{1}{L e} \frac{\alpha c}{\partial \xi}\right) \\
+(10)
\end{gathered}
$$

where the parameters $\alpha, \beta, \gamma, \mathrm{J}, \mathrm{U}^{*}$, and $\mathrm{V}^{*}$ are defined by

$$
\begin{aligned}
& \alpha=X_{\eta}^{2}+Y_{\eta}^{2}, \beta=X_{\xi} X_{\eta}+Y_{\xi} Y_{\eta}, \\
& \gamma=X_{\xi}^{2}+Y_{\xi}^{2}, J=X_{\xi} Y_{\eta}-X_{\eta} Y_{\xi} \\
& U^{*}=U Y_{\eta}-V X_{\eta}=\psi_{\eta}, V^{*}=V X_{\xi}-U Y_{\xi}=-\psi_{\xi}
\end{aligned}
$$

with the subscripts $\xi$ and $\eta$ standing for partial differentiation with respect to $\xi$ and $\eta$. Similarly, the boundary and initial conditions in $\xi \eta$-plane can be written as the following equations: 
at the surface of the cylinder

$\psi=0, \omega=\omega_{W A L L}, \theta=1, \gamma \frac{\partial c}{\partial \eta}-\beta \frac{\partial c}{\partial \xi}=0$

at the left and right walls

$\psi=0, \omega=\omega_{W A L L}, \theta=0, \gamma \frac{\partial c}{\partial \eta}-\beta \frac{\partial c}{\partial \xi}=0$

at top and bottom walls

$\psi=0, \omega=\omega_{W A L L}, \alpha \frac{\partial \theta}{\partial \xi}-\beta \frac{\partial \theta}{\partial \eta}=0, \alpha \frac{\partial c}{\partial \xi}-\beta \frac{\partial c}{\partial \eta}=0$

at central line

$\psi=0, \omega=0, \gamma \frac{\partial \theta}{\partial \eta}-\beta \frac{\partial \theta}{\partial \xi}=0, \gamma \frac{\partial c}{\partial \eta}-\beta \frac{\partial c}{\partial \xi}=0$

\section{initial condition}

$\psi=0, \theta=0, \omega=0, c=1-\frac{y}{B}$

where $\omega_{W A L L}$ is estimated as

$\omega_{W A L L}=-2 \frac{\psi_{C L}-\psi_{W A L L}}{L_{C L}^{2}}$

\section{3}

\section{Numerical method}

After generation of the grid meshes and calculation of the variables in Eq. (11), the governing Eqs. (7)-(10) along with the associated boundary conditions (12)-(17) were solved by using the finite difference method. The weighting function scheme [14] is adopted to discretize the governing equations. This scheme has been successfully used in solving the double-diffusive convection problem [9]. For grid generation method, it also has good performance [15]. Equations (8)-(10) can be expressed as the general form

$$
\begin{aligned}
J \frac{\partial \phi}{\partial \tau} & +\frac{\partial}{\partial \xi}\left(U^{*} \phi\right)+\frac{\partial}{\partial \eta}\left(V^{*} \phi\right)=\frac{\partial}{\partial \xi}\left(A \frac{\alpha}{J} \frac{\partial \phi}{\partial \xi}\right) \\
& +\frac{\partial}{\partial \eta}\left(A \frac{\gamma}{J} \frac{\partial \phi}{\partial \eta}\right)-\frac{\partial}{\partial \xi}\left(A \frac{\beta}{J} \frac{\partial \phi}{\partial \eta}\right) \\
& -\frac{\partial}{\partial \eta}\left(A \frac{\beta}{J} \frac{\partial \phi}{\partial \xi}\right)+S
\end{aligned}
$$

By using the weighting function scheme on equation for grid point $\mathrm{P}$ of an uniform grid system, one obtains the algebraic equation

$$
\begin{aligned}
a_{S W} \phi_{S W} & +a_{W} \phi_{W}+a_{N W} \phi_{N W}+a_{S} \phi_{S}+a_{P} \phi_{P} \\
& +a_{N} \phi_{N}+a_{S E} \phi_{S E}+a_{E} \phi_{E}+a_{N E} \phi_{N E}=a_{R}
\end{aligned}
$$

where

$$
\begin{aligned}
& a_{W}=\left(A \frac{\alpha}{J}\right)_{w} w_{f}\left(Z_{w}\right), a_{E}=\left(A \frac{\alpha}{J}\right)_{e} w_{f}\left(-Z_{e}\right) \\
& a_{S}=\left(A \frac{\gamma}{J}\right)_{s} w_{f}\left(Z_{s}\right), a_{N}=\left(A \frac{\gamma}{J}\right)_{n} w_{f}\left(-Z_{n}\right)
\end{aligned}
$$

$a_{p}=-a_{W}-a_{E}-a_{S}-a_{N}-\frac{J}{\Delta \tau}, a_{R}=-S-\frac{J \phi^{0}}{\Delta \tau}$

$a_{S W}=-2\left(A \frac{\beta}{J}\right)_{s w} w_{f}^{*}\left(Z_{s}\right) w_{f}^{*}\left(Z_{w}\right)$

$a_{N W}=2\left(A \frac{\beta}{J}\right)_{n w} w_{f}^{*}\left(Z_{n}\right) w_{f}^{*}\left(Z_{w}\right)$

$a_{S E}=2\left(A \frac{\beta}{J}\right)_{s e} w_{f}^{*}\left(Z_{s}\right) w_{f}^{*}\left(Z_{e}\right)$

$a_{N E}=-2\left(A \frac{\beta}{J}\right)_{n e} w_{f}^{*}\left(Z_{n}\right) w_{f}^{*}\left(Z_{e}\right)$

$Z_{w}=\left(\frac{U^{*} J}{A \alpha}\right)_{w}, Z_{e}=\left(\frac{U^{*} J}{A \alpha}\right)_{e}$

$Z_{s}=\left(\frac{V^{*} J}{A \gamma}\right)_{s}, Z_{n}=\left(\frac{V^{*} J}{A \gamma}\right)_{n}$

and the linear interpolating method is applied for calculating the quantity between the grid points, for example, $(A \alpha / J)_{w}=\left[(A \alpha / J)_{\mathrm{w}}+(A \alpha / J)_{P}\right] / 2$ and $2(A \beta / J)_{s w}=$ $(A \beta / J)_{S}+(A \beta / J)_{W}$, etc. The weighting function in the above equations can be calculated from the following equations [14].

$$
\begin{aligned}
W_{f}(Z) & =\left[0,(1-0.1|Z|)^{5}\right]+[0, Z], \\
W_{f}^{*}(Z) & =\left(2+0.3332 Z^{2}+0.0172 Z^{4}\right)^{-1}
\end{aligned}
$$

The stream function equation is somewhat different from the other equations. The first term in Eq. (18) is equal to zero and the last term becomes $\mathrm{J} \omega$.

For Eq. (7), the weighting factors are

$$
\begin{aligned}
& a_{W}=\left(\frac{\alpha}{J}\right)_{w}, a_{E}=\left(\frac{\alpha}{J}\right)_{e}, a_{S}=\left(\frac{\gamma}{J}\right)_{s}, a_{n}=\left(\frac{\gamma}{J}\right)_{n} \\
& a_{P}=-a_{W}-a_{E}-a_{S}-a_{N}, a_{R}=-J \omega \\
& a_{S W}=-\left(\frac{\beta}{J}\right)_{s w} / 4, a_{N W}=\left(\frac{\beta}{J}\right)_{n w} / 4 \\
& a_{S E}=\left(\frac{\beta}{J}\right)_{s e} / 4, a_{N E}=-\left(\frac{\beta}{J}\right)_{n e} / 4
\end{aligned}
$$

The values of $U^{*}$ and $V^{*}$ are equal to zero and thus $w_{f}(0)=1, w_{f}^{*}(0)=0.5$.

All the coefficients in the algebraic equations of the governing equation and boundary conditions are solved iteratively. The accuracy of iteration is defined as.

$\frac{M A X\left|\varphi^{k}-\varphi^{k-1}\right|}{M A X\left|\varphi^{k}\right|-M I N\left|\varphi^{k}\right|} \leq 10^{-5}$

where $\varphi$ indicates the physical variable of interest and superscript $\mathrm{k}$ denotes the iteration index.

In order to test the numerical method, the case of doublediffusive convection in a concentric cylinder was carried out. The comparison with the numerical and holographic interferometry results in [13] is shown in Fig. 2. The transient evolution of the flow field and transport phenomena, especially the refractive index distributions are examined. It is evident that they agree well. The grid mesh used in all 

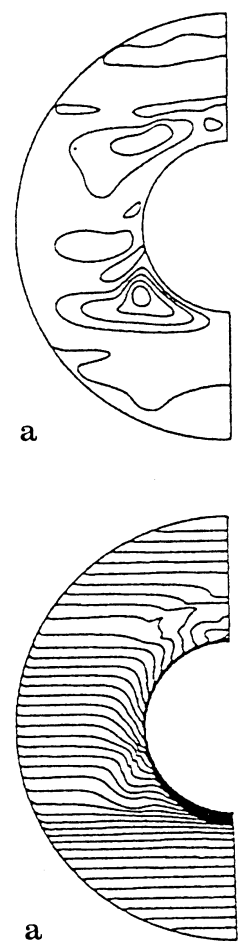
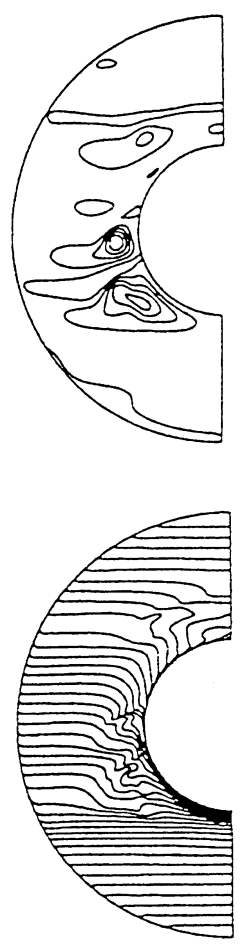
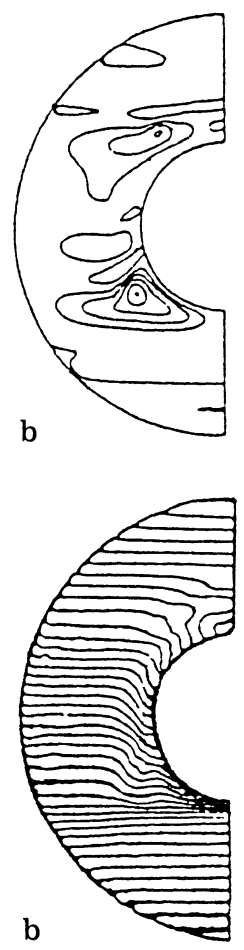

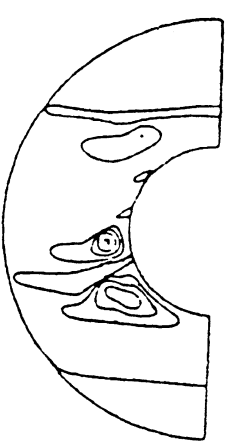

21

Fig. 2a, b. Comparison of the numerical and experimental results [13] for $R a_{T}=1.105 \times 10^{5}, R_{\rho}=15$. Figures at the upper column are stream lines of a present study, b prediction [13], figures at lower column are the refractive index of a present study, b experimental result [13]. Times are at $t=90 \mathrm{~s}$ and $t=120 \mathrm{~s}$, respectively

simulation is $231 \times 91$ and the grid distribution around the cylinder is very dense. This is because fine resolution near the layer interfaces is a necessity for accurate computations. The grid nodes around the cylinder are arranged the same as the concentric cylinder in previous comparison.

Due to the orthogonal boundary grids on the cylinder surface, the local Nusselt number $\mathrm{Nu}(\gamma)$ and the averaged Nusselt number, $\overline{\mathrm{Nu}}$ can be simply evaluated as follows:

$N u(\gamma)=-\partial \theta_{f} / \partial n ; \quad \overline{N u}=\frac{1}{2 \pi} \int_{0}^{2 \pi} N u(\gamma) d \gamma$

\section{4}

\section{Results and discussion}

The analysis was performed for a $\mathrm{H}_{2} \mathrm{O}-\mathrm{NaCl}$ solution. The values of the Prandtl number and Lewis number are fixed at 6.59 and 80 , respectively. Test cases at different thermal Rayleigh numbers, $R a_{T}=5 \times 10^{5}$ and $2 \times 10^{6}$, and at different buoyancy ratios, $R_{\rho}=4,2.67,1.33$, are discussed. Figures $3 a, 3 b, 3 c$ show the distributions of stream function, isotherms and isosolutal lines for $R a_{T}=5 \times 10^{5}$ and $R_{\rho}=2.67$ respectively. Most evident is the fact that, unlike the cases for unstratified fluid [16], there is suppression of plume development above the heated cylinder. From Fig. 3a, at $\tau=0.005$, it is shown that there are two vortices generated around the cylinder, as demonstrated in the experiments of $[10,12]$. The maximum stream function of the upper vortex is located at the top of the cylinder. The upper vortex is generated by the plume convection where the vertical upward temperature gradient interacts with the vertical downward salinity gradient. The warmer and salty fluid ascends to a height and then turns toward the sidewall and by interacting with the cooler and fresher ambient fluid, it finally descends to the top of cylinder and forms a vortex. When the vortex further erodes, it becomes a layer. This phenomenon is the same as that in the cases of heating the flat plate from below $[3,4]$. On the other hand, the lower vortex is at the lower half part of the cylinder. The bottom of the vortex is close to the bottom of the cylinder. This vortex is due to the onset of instability of lateral temperature gradient and vertical salinity gradients, as depicted in [5-9]. Due to the effect of heating, the warmer and salty fluid ascending along the cylinder is retarded by the cooler and fresher ambient fluid and flows toward the outer region. Finally the fluid flows to the cylinder and forms a vortex. While the fluid is flowing, the heat loses to the cold ambient fluid and the fluid parcel becomes weighty and, thus, makes the vortex intrusion slightly tilted, as seen at $\tau=0.005$. When the temperature of plume convection has further risen, the secondary flow pattern can be seen at the top of the cylinder, near the central line, as shown at $\tau=0.015$. This secondary flow is weaker than the primary vortex adjoining it but exists for a long time until it is compressed by the primary vortex. At $\tau=0.025$, at the upper part of the cylinder the vortex is also generated and becomes more and more significant. As discussed previously, the interface of the layers is also somewhat tilted.

From the contours before and after $\tau=0.015$, it is shown that the vortices generated around the lower half of the cylinder develop sooner than that at the upper half, and that they are smaller at the lower part of the cylinder, even though the heat effect is more obvious at the upper half part of the cylinder. This results from the different 
a
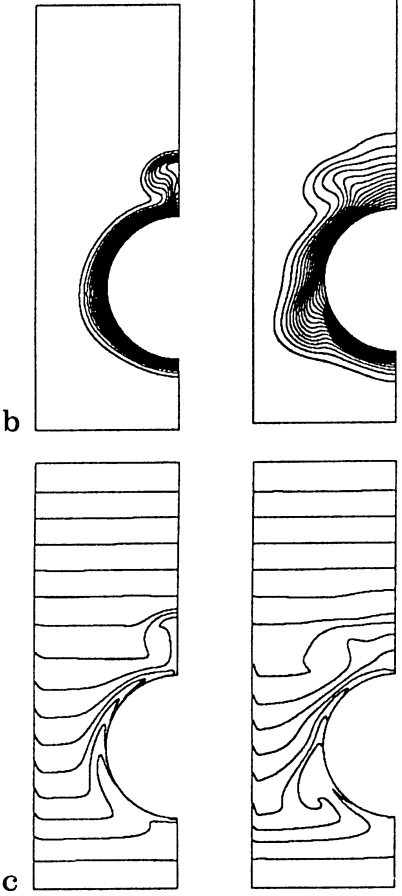

Fig. 3a-c. Predicted results of a stream lines, plots from left to right are $\Delta \psi=0.81,0.48,0.99,1.87 ; \mathbf{b}$ isotherms, where $\Delta \theta=0.0625$ for all cases; $\mathrm{c}$ isosolutal lines, where $\Delta c=0.0625$ for all cases. The parameters are $R a_{T}=5 \times 10^{5}, R_{\rho}=2.67$ and times from left to right are $\tau=0.005,0.015,0.025,0.045$, respectively

effects of the salinity gradient prior to the onset of stability, as depicted in $[8,9]$. The no-diffuse boundary condition at the cylinder makes the isosolutal lines deflect upward adjoining the lower half of the cylinder and downward adjoining the upper half of the cylinder. The salinity gradient near the upper half of the cylinder is negative in the horizontal direction such that there is a tendency of fluid to move downward. The salinity gradient near the lower half of cylinder is positive in horizontal direction such that there is a tendency of fluid to move upward. When the cylinder is heated, the thermally induced fluid moves upward along the cylinder. The thermally driven force and the solutally driven force have the same direction near the lower half of the cylinder such that the onset of instability is sooner. In contrast, the thermally driven force and the solutally driven force have opposite direction near the upper half of the cylinder such that the onset of instability is slower. This also leads to a larger layer at the lower half of the cylinder. There are three obvious convection layers can be seen at $\tau=0.025$. The first layer is calculated from the top, generated above the basic plume and should belong to the first type, as shown in Fig. 3a, at $\tau=0.015$. Because the layer is weak compared to the other layers, it is not demonstrated on the later figures. The layer generated due to basic plume is a type two layer. The other layers below the second type and around the cylinder are of the third type.

For the temperature distribution, when the layer is generated, the S-shape distribution can be seen at the second and third types of layers, as shown in Fig. 3b. This is a typical characteristic of a double-diffusive layered structure subject to lateral heating $[5,9]$. The whole distribution of temperature is composed of the plume convection and the layers around the cylinder, which is consistent with experimental results $[11,12]$. The heat transfer in layers is convection dominated while that at the interface is conduction. When the layers are progressing, heat is not only transferred from the cylinder, but also between the layers. The directions of heat transfer are from the lowest layer and the highest layer toward to the layers at middle region. This tendency is similar to the case of lateral heating in a rectangular cavity. However, in both cases, it happens for different reasons. The top and bottom layers grow first due to the constraint of top and bottom plates [7, 9]. In this study, the convection of layers is noticeable at the top and bottom layers due to the plume convection and the earlier onset of instability at lower half of the cylinder. On the other hand, there is a better mixed concentration distribution in the layers and a sharp difference at the interface, as shown in Fig. 3c. Outside the layer region, the salinity distribution is still linear.

The flow field, temperature and concentration distributions at a higher buoyancy ratio, $R_{\rho}=4$, are shown in Fig. $4 \mathrm{a}, 4 \mathrm{~b}, 4 \mathrm{c}$, respectively. The height of the layer due to plume convection is lower than that for the case of $R_{\rho}=2.67$. This is because the increase in salinity difference retards the plume convection. Besides, all of the layers are weaker than those of the previous case. However, the interaction of the vertical salinity gradient and the horizontal temperature gradient is reinforced and the onset of instability is also sooner than that at the case of $R_{\rho}=2.67$. In addition, the cell numbers also increase.

Four vortices can be seen at $\tau=0.025$. It is interesting to note that the second vortex calculated from the top is not stable. This vortex is generated last and is compressed by the vortices above and below it. This weak vortex disappears first as the flow field evolves. The second and third types of layers can also be seen clearly in the flow field and the heat and mass transfer rate are also lower than the case of $R_{\rho}=2.67$, as shown in Fig. $4 \mathrm{~b}$ and $4 \mathrm{c}$. The variation of temperature distribution in the weak layer is not as significant as in other layers.

On the other hand, when the buoyancy ratio decreases, $R_{\rho}=1.33$, the flow motion is easier and more vigorous, as seen in Figs. $5 a, 5 b, 5 c$ and the plume convection is stronger than in the previous two cases. However, since the interaction of thermal and solutal gradients decreases, the onset of instability is delayed, and the layer number around the cylinder is decreased. The layer at the upper half of the cylinder cannot be generated and is replaced by 

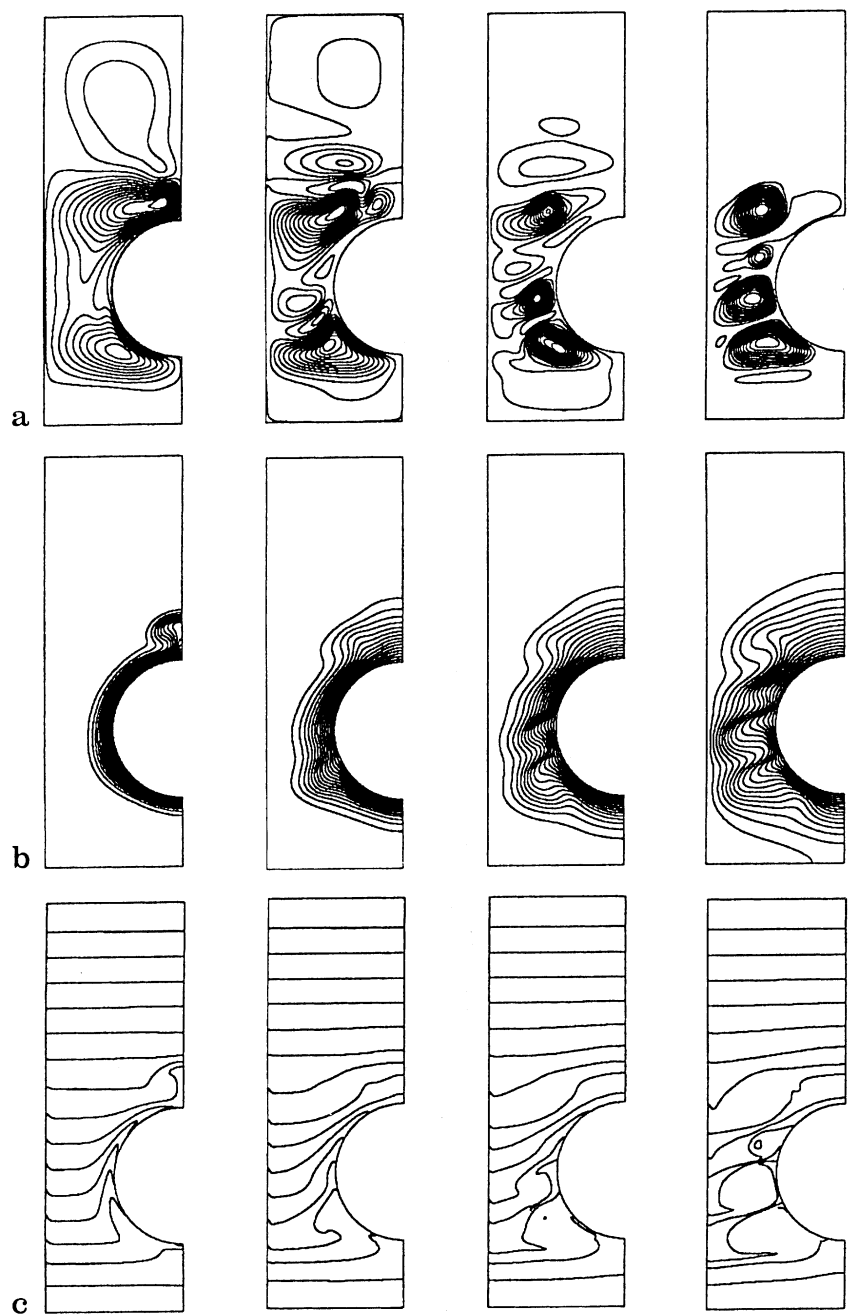

Fig. 4a-c. Predicted results of a stream lines, plots from left to right are $\Delta \psi=0.62,0.24,0.28,0.53 ; \mathbf{b}$ isotherms, where $\Delta \theta=0.0625$ for all cases; $\mathrm{c}$ isosolutal lines, where $\Delta c=0.0625$ for all cases. The parameters are $R a_{T}=5 \times 10^{5}, R_{\rho}=4$ and times from left to right are $\tau=0.005,0.015,0.025,0.045$, respectively

the layer generated from lower half, as seen at $\tau=0.015$. It is noted that if the thermally driven force is larger, for the same solutally driven flow, the convection is significant. This benefits the generation of the layers due to plume convection (like type one and type two) but has the opposite effect on the generation of layers of third type. In contrast, if the solutally driven force is larger, for the same thermally driven force, the convection is retarded and generation of the layers due to plume convection (like type one and type two) is suppressed. This benefits the generation of layers of the third type. The temperature and salinity distributions also demonstrate the more vigorous convection in the flow field. This vigorous convection improves the heat transfer and increases the significance of well-mixed region.

Figures $6 \mathrm{a}, 6 \mathrm{~b}$ and $6 \mathrm{c}$ are the stream functions for $R a_{T}=2 \times 10^{6}$ and $R_{\rho}=4,2.67,1.33$, respectively. Comparing the results between Fig. 6a and Fig. 3, it is shown that convection is more noticeable, and that the layers evolve sooner. Although the thermally driven flow increases significantly, the plume development can not
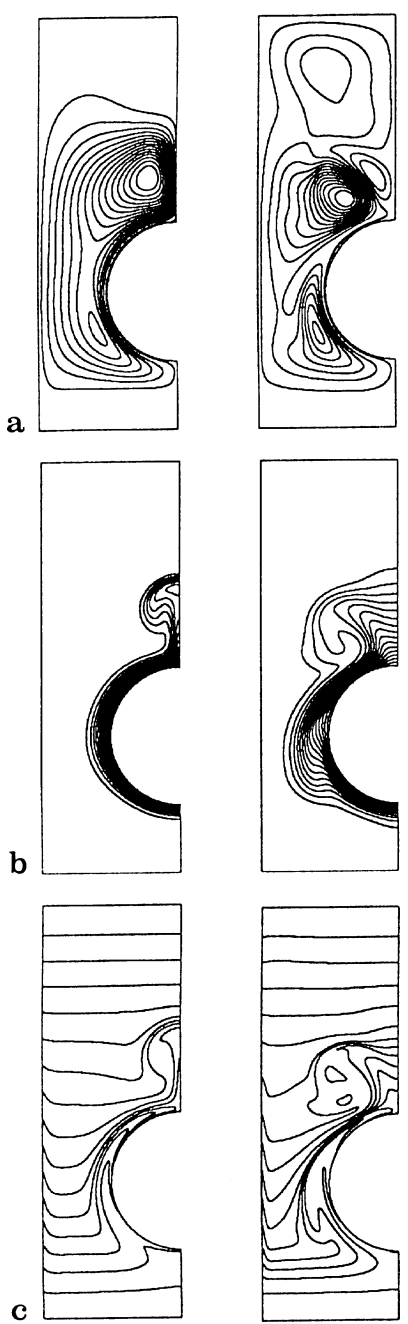
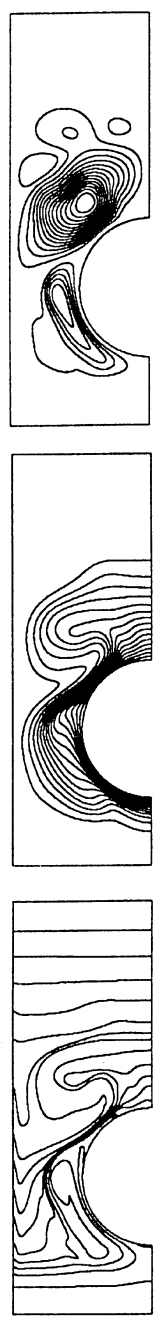

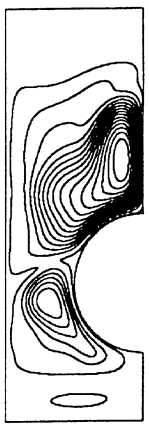

23
Fig. 5a-c. Predicted results of a stream lines, plots from left to right are $\Delta \psi=1.69,1.42,1.71,2.32$; b isotherms, where

$\Delta \theta=0.0625$ for all cases; $c$ isosolutal lines, where $\Delta c=0.0625$ for all cases. The parameters are $R a_{T}=5 \times 10^{5}, R_{\rho}=1.33$ and times from left to right are $\tau=0.005,0.015,0.025,0.045$, respectively

sufficiently ascend to the higher region. This is because the suppression due to salinity gradient also increases. The interaction of thermal and solutal buoyancy forces is of such significance that the onset of instability is soonest. There are four layers at $\tau=0.015$. It is interesting that the evolution of the fluid field is similar to that of Fig. 4. Similar comparisons can be made between Fig. $6 \mathrm{~b}$ and Fig. 3 and Fig. $6 \mathrm{c}$ and Fig. 5. There are three layers at $\tau=0.015$ for Fig. $6 \mathrm{~b}$. The secondary vortex at the top of cylinder is weaker than that for $R a_{T}=5 \times 10^{5}$ and it disappears sooner. When the buoyancy ratio decreases the thermally driven force is stronger and the solutally driven force is weaker. This leads to an earlier onset of instability, as shown in Fig. 6c. The layer caused by the basic plume is merging with the layers generated around the cylinder at $\tau=0.025$. These phenomena can also be seen in the results of shadowgraph experiments in [12] and from numerical simulation the transport phenomena can be realized more clearly. It is worth noticing that the first type of layer is also more vigorous, and the interface can be seen clearly above the basic plume at $\tau=0.045$, as shown in Fig. 7a, 

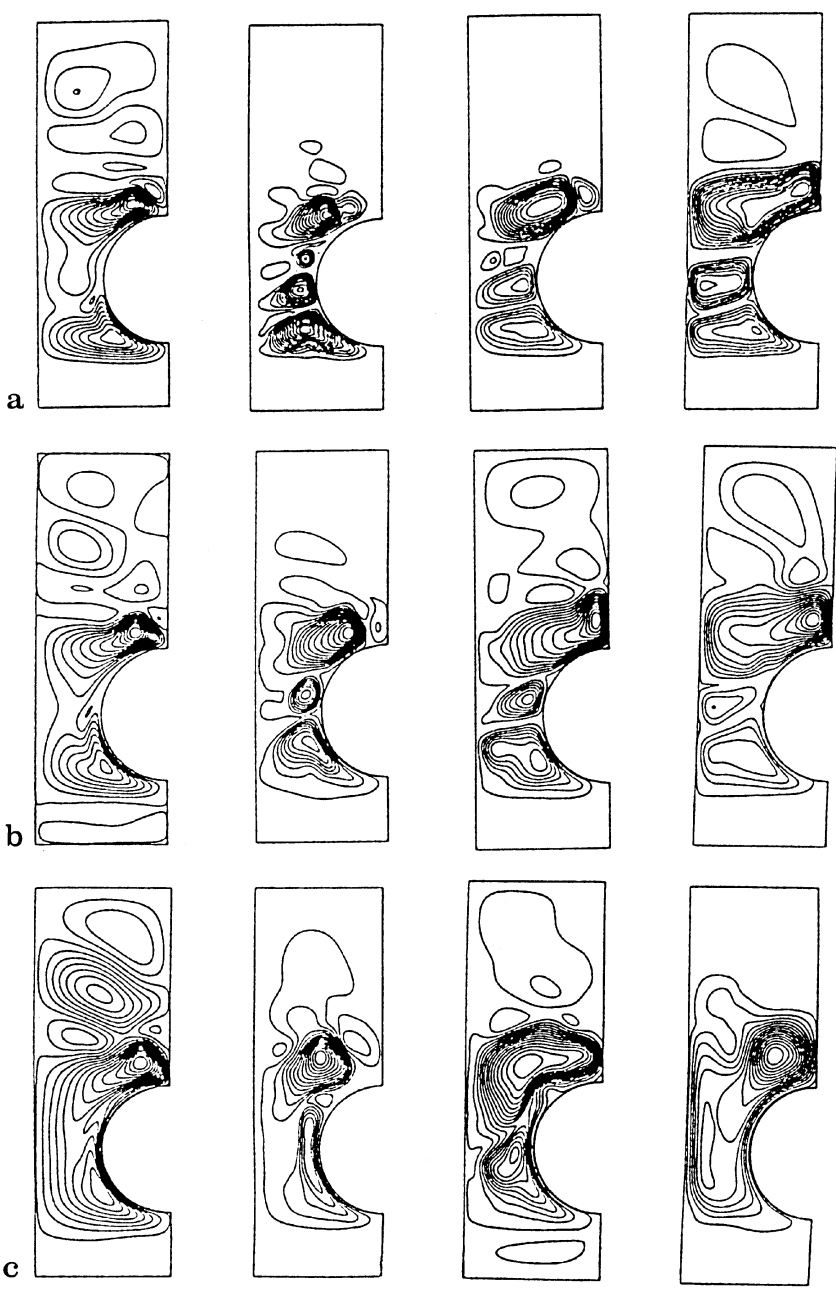

Fig. 6a-c. Predicted stream lines for $R a_{T}=2 \times 10^{6}$ and $\mathbf{a} R_{\rho}=4$, plots from left to right are $\Delta \psi=0.89,0.89,2.1,1.7$;

b $R_{\rho}=2.67$, plots from left to right are $\Delta \psi=1.44,1.9,2.27$, 3.9 ; c $R_{\rho}=1.33$, plots from left to right are $\Delta \psi=2.07,2.69$, $2.51,6.52$. The times from left to right are $\tau=0.005,0.015$, $0.025,0.045$, respectively

7b. This indicates that the temperature and solutal distributions of the first type of layer are different from the other two types of layers. They are similar to the case of heating from below [4]. It is noted that the plume development in all of above cases is not noticeable as in the experiments on constant heat flux [10-12]. This prevents the layers around the basic plume from being generated. Only the layers generated due to basic plume appear. Besides, the layer formation of the first type is not as significant as in experiments [10-12].

Figures $8 \mathrm{a}, 8 \mathrm{~b}$, and $8 \mathrm{c}$ describe the local Nusselt number at different times for the parameter $R a_{T}=5 \times 10^{5}$. The values are predicted from top $\left(\gamma=0^{\circ}\right)$ to bottom $\left(\gamma=180^{\circ}\right)$. The plots of local Nusselt number at different angles for $R a_{T}=5 \times 10^{5}$ and $R_{\rho}=4$ are shown in Fig. 8a. At $\tau=0.015$, the local Nusselt number is at a minimum at the upper stagnation point. The rises and falls can be seen in the plot. These are due to the two vortices around the cylinder and the variation is more significant during the flow evolution. The layer structure gives the wave plot
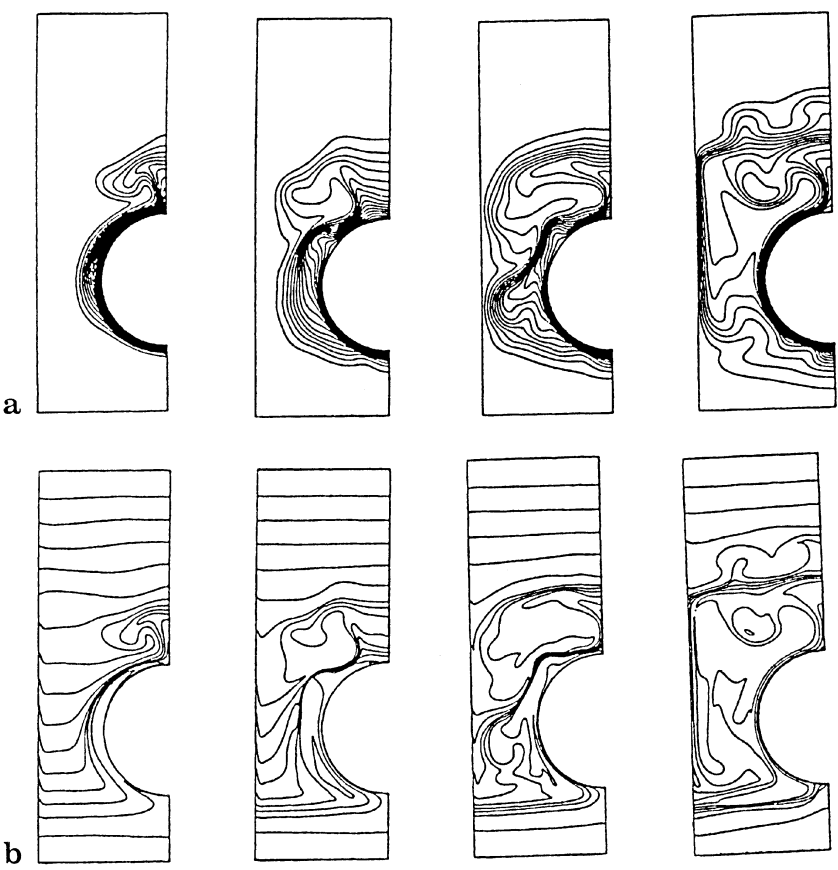

Fig. 7a, b. Predicted results of $\mathbf{a}$ isotherms, where $\Delta \theta=0.0625$ for all cases; b isosolutal lines, where $\Delta c=0.0625$ for all cases. The parameter is $R a_{T}=2 \times 10^{6}, R_{\rho}=1.33$ and the times from left to right are $\tau=0.005,0.015,0.025,0.045$, respectively

distribution, as depicted in $[7,9]$. Since secondary flow is generated, the heat transfer is retarded and the distributions at the upper part of the cylinder decrease with time. From Fig. $8 \mathrm{~b}$ it can be seen that the more significant variation is at upper part of the cylinder due to the fact that the secondary flow is weaker than that at Fig. 8a. Also, the primary vortices can directly effect the heat transfer characteristics. When the buoyancy ratio decreases further, convection is more vigorous causing the local Nusselt number to increase. Since there are only two vortices, the related rises and falls can be seen in plots. The plume convection is more and more vigorous and the lower vortex is weaker and weaker during its time of evolution causing the local Nusselt number at the upper half of the cylinder to increase and that at the lower part to decrease. The locations of the minimum are near $\gamma=90^{\circ}$ in Figs. 8a, $8 \mathrm{~b}$ and $8 \mathrm{c}$ and move forward as buoyancy ratio increases. It is shown that the size of lower layer increases with decreasing buoyancy ratio. The variation is not as significant as at the upper part of the cylinder.

The typical temporal variation of averaged Nusselt numbers at different $R_{\rho}$ are shown in Fig. 9. From Fig. 9, it is found that the heat transfer characteristics of doublediffusive convection are between natural convection and conduction, as also shown in [13]. The averaged Nusselt number first decreases sharply and then the decrease is more gradual. The layer structure leads to the variation of the distribution and this is also different from both modes of natural convection and conduction. Because the linear solutal gradient is imposed initially, the solutal Rayleigh number also represents the degree of stratification. For the same thermal Rayleigh number, larger buoyancy ratio 


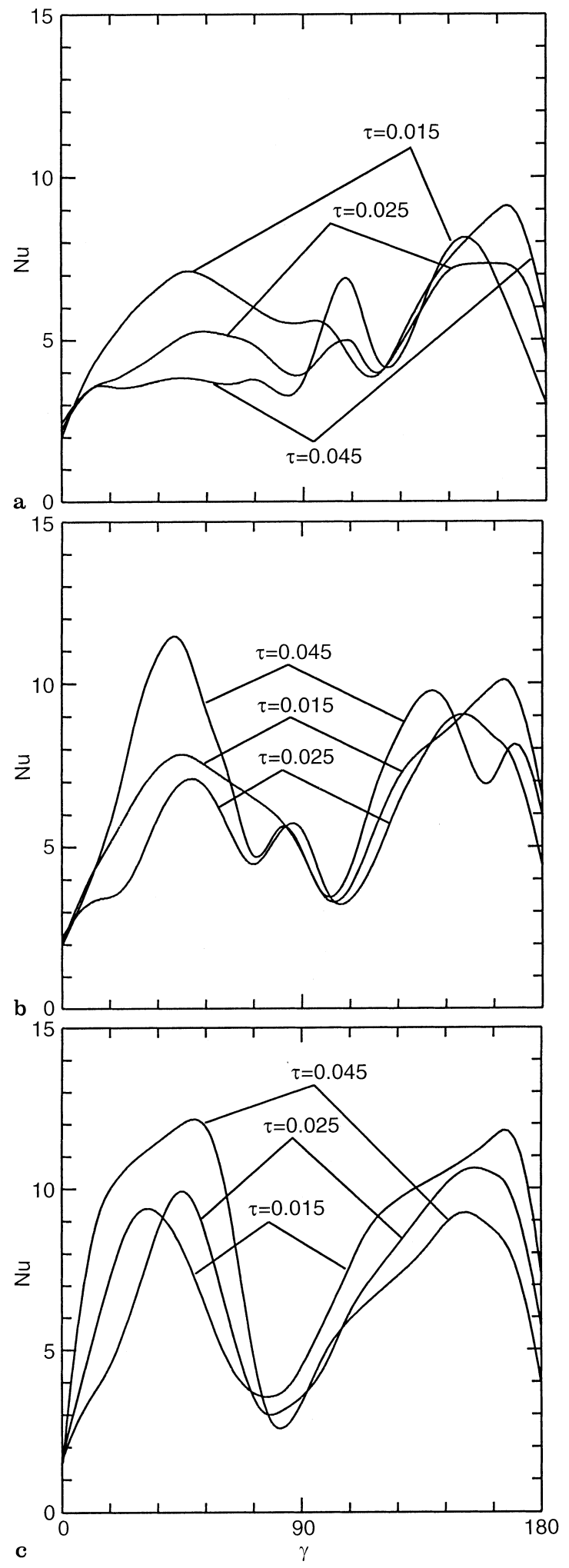

Fig. 8a-c. Plots of local Nusselt number at different time for $R a_{T}=5 \times 10^{5}$ and $\mathbf{a} R_{\rho}=4 ; \mathbf{b} R_{\rho}=2.67$; $R_{\rho}=1.33$

leads to a slower flow motion. The averaged Nusselt number at $R a_{T}=2 \times 10^{6}$ is higher than that shown in Fig. 9 due to the stronger thermal buoyancy force, as also described in [9].

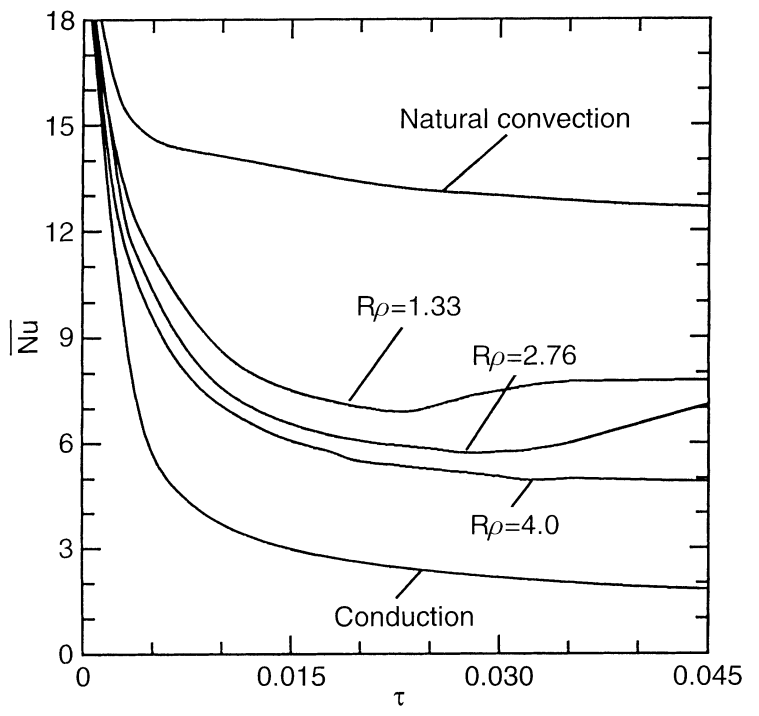

Fig. 9. Plots of average Nusselt number with respect to time for $R a_{T}=5 \times 10^{5}$

5

\section{Conclusion}

Double-diffusive convection due to a salt-stratified fluid layer with a cylindrical heat source in a rectangular cavity is numerically investigated, using the body-fitted grid generation method. The nature of flow and heat transfer strongly depends on the thermal Rayleigh number and buoyancy ratio. The layers generated at the top of basic plume are classified as the first type and are caused by plume convection. The onset of instability is due to the interaction of the upward temperature gradient and downward salinity gradient and the layers are subject to the heating effect from below. These layers, due to basic plume, are of the second type and the onset of instability is due to the interaction of the upward temperature gradient and downward salinity gradient. The layers are subject to heat from a lateral direction. The layers generated around the cylinder are of the third type and, in this case, the onset of instability is due to the interaction of the lateral temperature gradient and the downward salinity gradient. This layer is also subject to lateral heating.

The first onset of instability is at the lowest half of the cylinder and the top of the cylinder. The no-diffuse solutal boundary condition around the cylinder leads to thermally and solutally driven buoyancy forces in the same direction at the lower half of the cylinder and in the opposite direction at the upper half. The onset of instability is earlier at the lower half than that at the upper, even though there is a more significant heating effect at the upper. For the same thermal Rayleigh number, the increase in buoyancy ratio retards the onset of layer instability due to plume convection but reinforces the interaction of temperature and salinity gradients at side of the cylinder. In addition, for the same thermal Rayleigh number, the layer numbers increase with the buoyancy ratio. However, the convection in the layers is weakened due to the increase in buoyancy ratio. When the thermal Rayleigh number increases, the convection is more vig- 
orous for the same buoyancy ratio and the interaction of the temperature and salinity gradients are also more obvious. The layer formation and evolution come sooner. For the second and third types of layers the temperature distributions are similar. The S-shaped distribution can be seen in the contours. However, the temperature distribution of the first type is different from the other two types of layers because it results from heating from below. The distributions of salinity are similar for all types of layers as the well mixed region can be seen in the layers while sharp salinity difference can be found at the interface.

The local Nusselt number reflects the variation of layer structure in the flow field. The convection in layers leads to a high rate of heat transfer from the cylinder and forms the values of peaks. However, the heat transfer is retarded by the interface and is, thus, reduced. When the buoyancy ratio decreases, the convection in the flow field is reinforced so that the local Nusselt number increases and the variation decreases. The averaged Nusselt number varies with time and is between both the conduction and natural convection modes. Since there is the complex variation due to layer structure, the averaged Nusselt number does not smoothly decay as in these two modes.

\section{References}

1. Turner, J. S.: Buoyancy effects in fluids. Cambridge University Press, 1973

2. Ostrach, S.: Natural convection with combined driving forces. PhysicoChem. Hydrodyn. 1 (1980) 233-247

3. Huppert, H. E.; Linden, P. F.: On heating a salinity gradient from below. J. Fluid Mech. 95 (1979) 431-464

4. Kazmierczak, M.; Poulikakos, D.: Transient double diffusion in a layer heated from below. Int. J. Heat Fluid Flow 11 (1990) 30-39
5. Thorpe, S. A.; Hutt, P. K.; Soulsby, R.: The effect of horizontal gradients on the thermohaline convection. J. Fluid Mech. 38 (1969) 375-400

6. Chen, C. F.; Briggs, D. G.; Writz, R. A.: Stability of thermal convection in a salinity gradient due to lateral heating. Int. J. Heat Mass Transfer 14 (1971) 57-65

7. Lee, J. W.; Hyun, J. M.: Time dependent double diffusion in a stabely stratified fluid under lateral heating. Int. J. Heat Mass Transfer 34(1991) 2409-2421

8. Thangam, S.; Zebib, A.; Chen, C. F.: Double-diffusive convection in an inclined fluid layer. J. Fluid Mech. 116 (1982) 363-378

9. Chen, Y. M.; Liu, C. K.: Time dependent double-diffusive convection due to salt-stratified fluid layer with differential heating in an inclined cavity. Int. J. Heat Mass Transfer 40(1997) 711-725

10. Hubbell, R. H.; Gebhart, B.: Transport processes induced by a heated horizontal cylinder submerged in quiescent salt-stratified water. Proc. Heat Transfer and fluid Mechanics Institute (1974) 203-219

11. Tsinober, A. B.; Yahalom, Y.; Shlien, D. J.: A point source of heat in a salinity gradient. J. Fluid Mech. 135 (1983) 199-217

12. Neilson, D. G.; Incropera, F. P.: Double diffusive flow and heat transfer for a cylindrical source submerged in a salt-stratified solution. Int. J. Heat Mass Transfer 30 (1987) 2559-2570

13. Dosch, J.; Beer, H.: Numerical simulation and holographic visualization of double diffusive convection in a horizontal concentric annulus. Int. J. Heat Mass Transfer 35 (1992) $1811-1821$

14. Hsu, K.; Lee, S. L.: A numerical technique for two-dimensional grid generation with grid control at all of the boundaries. J. Comput. Phys., 96 (1991) 451-469

15. Chiou, C. C.; Lee, S. L.: Forced convection on a rotating cylinder with a inclined air jet. Int. J. Heat Mass Transfer 36 (1993) 3841-3850

16. Incropera, F. P.; Yaghoubi, M. A.: Buoyancy driven flows originating from heated cylinders submerged in a finite water layer. Int. J. Heat Mass Transfer 23 (1980) 269-278 